\title{
A comparative survey of small RNA and their targets in grapevine embryogenic callus cultures and young leaves
}

D. Lizamore ${ }^{1}$ and C. Winefield ${ }^{1}$

${ }^{1}$ Dept. Wine, Food and Biomolecular Sciences, Lincoln University, Lincoln, New Zealand

\begin{abstract}
Plant embryogenic callus (EC) cultures are important both for clonal propagation and as a source of explant material for transgenesis experiments. In grapevine, much work has been done to develop protocols for establishing EC cultures from multiple varieties. However, tissue culture provides a stress effect that can alter both to the genome, through the activation of transposable elements, and the epigenome. The host cell response to genomic stress is two-fold: post-transcriptional gene silencing (PTGS) targets aberrant transcripts for cleavage and sequestration, and transcriptional gene silencing (TGS) targets specific DNA loci for RNA-dependent DNA methylation (RdDM) and histone modifications. Small RNA molecules direct these systems to their targets based on sequence complementarity. To better understand silencing activity in EC cultures, we undertook a survey molecules of small from these cultures using parallel short read sequencing and compared these with small RNA from young leaf tissue. Our results show that small RNA profiles differ greatly among the two tissue types. One third of the small RNAs found in leaf were known microRNA (miRNA) sequences that regulate the development of vegetative tissue. MicroRNAs were also present in EC, but comprised less than one per cent of the small RNA complement. Comparison of the gene ontology (GO) networks of target genes for each sample revealed complex and distinct patterns of gene regulation in each tissue type. Trans-acting siRNAs (tasiRNAs) from 18 loci were also present at over 100 reads per million (RPM) in leaf, all of which were decreased or absent in EC. In contrast, EC contained approximately twice the amount of $24 \mathrm{nt}$ small interfering RNAs (siRNAs) associated with maintaining the repression of repeat-associated DNA as was found in the leaf tissue. The results indicate that not only do the targets of RNA interference (RNAi) vary in EC with respect to leaf tissue, but that the levels of activity for the various silencing systems differ greatly. Researchers studying plants recovered through somatic embryogenesis would be advised to take these observations into consideration, particularly when the introduction of foreign DNA is involved.
\end{abstract}

Keywords: siRNA, microRNA, grapevine, epigenetics, somatic embryogenesis, silencing, tasiRNA

\section{INTRODUCTION}

Embryogenic callus (EC) cultures are a type of totipotent tissue frequently produced and maintained for the clonal propagation of various plant species by somatic embryogenesis. In grapevine, EC are typically used as explant material for transformation, a task for which this tissue type has proved most suitable despite low efficiencies and inter-varietal variation (Dhekney et al., 2009). Most such experiments include the assumption that the genome remains immutable when cultured in this way, despite common observations of somaclonal variation (Kuksova et al., 1997; Schellenbaum et al., 2008). It is also known that tissue culture, along with other stresses, affects the profile of a plant's epigenome, with consequences that include the release of transposable element (TE) silencing (Slotkin and Martienssen, 2007). Such epigenetic changes are likely to be accompanied by altered abundances of small RNAs, which serve to guide for RNA interference (RNAi) complexes involved in both post-transcriptional gene silencing (PTGS) and RNA-dependent DNA methylation (RdDM).

Eukaryotic cells have evolved systems to prevent the genotoxic effects of viruses and TEs that are dependent on small RNAs for target identification (Lisch and Bennetzen, 2011). These defence systems oppose the successful incorporation of foreign nucleic acid sequences into the genome and can regulate the subsequent epigenetic status of novel DNA (Slotkin and Martienssen, 2007). Therefore, knowledge of the small RNA environment within EC cultures is a crucial step towards understanding the genetic and epigenetic consequences of somatic embryogenesis on vines and the use of this tissue as a target for grapevine transformation. 
The small RNA molecules within a cell can be distinguished both by their length and functionality. MicroRNAs (miRNAs) are typically 21-22 nucleotide (nt) fragments produced by the cleavage of hairpin secondary structures of pre-miRNA transcripts with inverted repeat regions by the DICER-Like1 (DCL1) protein complex. One strand of a miRNA pair will associate with ARGONAUTE1 (AGO1) in the RNAinduced silencing complex (RISC) to target mRNA transcripts, based on imperfect binding, which are cleaved or to inhibited from translation (Voinnet, 2009).

Small interfering RNAs (siRNAs), which guide both PTGS and RdDM silencing complexes, are produced by the processing of double stranded RNA (dsRNA). Heterochromatic siRNAs (hc-siRNAs) are generated from RNA Polymerase IV (Pol IV) transcripts of heterochromatic DNA, following complementary strand synthesis by RNA-dependent RNA polymerase 2 . The dsRNA are cleaved into 2324 nt siRNAs by DICER-like 3 and proceed to direct RdDM of perfectly matched sequences across the genome.

Trans-acting siRNAs (tasiRNAs) are produced from transcripts of non-coding TAS loci, which are cleaved by miRNA-loaded AG01 proteins. The remaining transcript is made double-stranded by RdR6 and cleaved into phased $21 \mathrm{nt}$ small RNAs by DL2 and DCL4. TasiRNAs loaded into AGO1 and play a role in the normal growth and development of tissues through the regulation of target gene transcripts. These same four enzymes are also known to produce $21 \mathrm{nt}$ siRNAs from TEs when they are transcribed by RNA Pol II.

Here we present data from parallel sequencing of small RNA reads from both EC and young leaf tissue of Pinot noir clone UCD5. By sorting the reads into functional small RNA categories, we are able to make comment on the commonalities and differences among the targets of silencing in these two tissue types.

\section{MATERIALS AND METHODS}

\section{Sample preparation and sequencing}

Six young opened leaves (less than $30 \mathrm{~mm}$ diameter) from Vitis vinifera cv. Pinot noir clone UCD5 were ground together in liquid nitrogen. Likewise, EC cultures of the same genotype growing on $\mathrm{C}^{1} \mathrm{P}$ medium (Torregrosa, 1998)from three Petri plates were ground to powder. Small RNA was purified from $50 \mathrm{mg}$ aliquots of each sample using the Plant microRNA Purification Kit (Norgen Biotek, Canada). RNA fragments 18-35 nt in size were selected by polyacrylamide gel electrophoresis and used by New Zealand Genomics Ltd to prepare a TruSeq Small RNA sequencing library. The two libraries were combined on one lane of an Illumina MiSeq flow cell and sequenced using $50 \mathrm{bp}$ single-end sequencing.

Adapter trimming and size filtering of fragments was performed in the UEA small RNA Workbench (Stocks et al., 2012). Reads that mapped to Vitis vinifera tRNA and rRNA from RFAM (Nawrocki et al., 2015) were removed using Patman (Prüfer et al., 2008).

\section{miRNA analysis}

MicroRNA sequences from miRBase version 21 (Kozomara and Griffiths-Jones, 2014) and novel potential miRNAs were identified using miRCat (Stocks et al., 2012). Predicted miRNAs were filtered for the presence of a miRNA* sequence, minimum free energy (MFE) less than -28, and GC content between $33 \%$ and $62 \%$ as recommended by Meyers et al. (2016). Read counts were normalised to the total number of small RNA reads per library (after tRNA and rRNA filtering) in millions.

To analyse the activity of miRNAs most actively contributing to gene regulation, only those with more than 100 reads per million (RPM) in either sample were analysed further. MicroRNA targets were predicted using the psRNATarget algorithm (Dai and Zhao, 2011), by comparing each miRNA sequence with the Vitis vinifera CRIBI V2.1 transcript set (Vitulo et al., 2014). A maximum expectation of 5.0 was used as this has been found to be necessary for the identification of regulated genes (McCue et al., 2013). For each target gene set, overrepresented gene ontologies (GOs) were determined by comparing the list of target genes with the complete Vitis vinifera transcriptome set using FatiGO+ (Al-Shahrour et al., 2004). Enriched gene ontologies were then summarised and network diagrams created using REVIGO comparison with the Arabidopsis thaliana database at a similarity score of 0.5 (Supek et al., 2011).

\section{siRNA analysis}

Trans-acting siRNAs were identified by searching for 21 nt phased reads using the tasiRNA prediction algorithm developed by Chen et al. (2007) as implemented in the UEA Small RNA Workbench 
(Stocks et al., 2012). Loci producing phased $21 \mathrm{nt}$ degradation products from each library were combined to create a single list, with overlapping features identified in both libraries extended to define a single fulllength tasiRNA gene locus. Total phased reads at each locus were counted for each library and normalised to the total number of small RNA reads in millions (after miRNA filtering).

\section{RESULTS AND DISCUSSION}

\section{Read filtering and sorting}

Small RNA sequencing produced over 7 million reads per library (Table 1). Although the EC library had fewer reads, sequence diversity was higher, with almost twice as many (185\%) distinct reads. Similar read proportions were removed by the quality control filtering steps for each library, but the leaf tissue library consisted of far more miRNA reads (25.9\%) than the EC library $(0.4 \%)$.

Table 1. Sequence reads remaining after processing.

\begin{tabular}{|c|c|c|c|c|}
\hline \multirow{2}{*}{ Processing stage } & \multicolumn{2}{|c|}{ Young leaves } & \multicolumn{2}{|c|}{ Embryogenic callus } \\
\hline & Total reads & Distinct reads & Total reads & Distinct reads \\
\hline Sequenced reads & $7,886,332(100 \%)$ & $1,238,982(100 \%)$ & $7,010,188(100 \%)$ & $2,294,748(100 \%)$ \\
\hline 3' adaptor removal & $7,633,928(97 \%)$ & $1,009,121(81 \%)$ & $6,780,664(97 \%)$ & $1,977,933(86 \%)$ \\
\hline Filter by length & $6,738,881(85 \%)$ & 936,553 (76\%) & $4,842,717$ (69\%) & 1,821,213 (79\%) \\
\hline Filter tRNA / rRNA & $4,859,237(62 \%)$ & 898,097 (72\%) & $3,439,527$ (49\%) & $1,776,990(77 \%)$ \\
\hline Filter to remove miRNA & $2,818,653(36 \%)$ & $898,012(72 \%)$ & $3,411,631(49 \%)$ & $1,776,928(77 \%)$ \\
\hline
\end{tabular}

\section{Micro RNAs}

The two tissue types showed very different profiles with respect to miRNA abundance. Embryogenic callus-derived miRNAs included 12 sequences that were present at concentrations above 100 reads per million (RPM), a threshold that has been suggested as a lower limit for potential silencing activity based on experimentally verified functional miRNAs (McCue et al., 2013).

Eleven sequences from the young leaf library were above this threshold, but three of these are from the highly abundant vvi-miR166 family, which accounted for a combined 333,445 RPM (Table 2). The abundance of vvi-miR166 accounts for the large difference in total miRNA content between the two tissues. miR166 is a well conserved microRNA among land plants and is know to be associated with shoot apical meristem development and specification of leaf polarity (Kidner and Martienssen, 2004). In contrast, EC libraries contained a high proportion of miR156 and mir171 reads, associated with embryogenesis in Arabidopsis and sweet orange respectively (Nodine and Bartel, 2010; Wu et al., 2011). EC also had high levels of miR159, not seen in leaf, which negatively regulates GAMYB genes that inhibit growth and promote programmed cell death.

Interestingly, miR2118 and miR482 were mutually exclusive among the two tissues, although both are members of the same superfamily of miRNAs, which are known to suppresses the activity of defence response genes in the absence of a pathogen attack in Solanum species (Shivaprasad et al., 2012). This difference may indicate that the two tissue types are differentially primed for disease response.

Table 2. Normalised abundance of miRNA sequences present at over 100 reads per million (RPM) in small RNAs from either young leaf or EC tissue and the number of predicted gene targets for each.

\begin{tabular}{llllll}
\hline Name & miRNA sequence & Length & Leaf RPM & EC RPM & Gene targets \\
\hline vvi-miR156b & UGACAGAAGAGAGUGAGCAC & 20 & 1 & 956 & 94 \\
vvi-miR159c & UUUGGAUUGAAGGGAGCUCUA & 21 & 0 & 1,072 & 100 \\
vvi-miR162 & UCGAUAAACCUCUGCAUCCAG & 21 & 621 & 109 & 66 \\
vvi-miR166a-1 & UCUCGGACCAGGCUUCAUUCC & 21 & 58,481 & 697 & 65 \\
vvi-miR166b-1 & UCGGACCAGGCUUCAUUCCUC & 21 & 332 & 1 & 68 \\
vvi-miR166c & UCGGACCAGGCUUCAUUCCCC & 21 & 274,632 & 388 & 67 \\
vvi-miR171f & UUGAGCCGCGCCAAUAUCACU & 21 & 0 & 358 & 72 \\
\hline
\end{tabular}




\begin{tabular}{llllll}
\hline vvi-miR2118 & UUGCCGACUCCACCCAUACCUA & 22 & 276 & 0 & 86 \\
vvi-miR319b-1 & CUUGGACUGAAGGGAGCUCCC & 21 & 590 & 0 & 85 \\
vvi-miR319b-2 & UUGGACUGAAGGGAGCUCCC & 20 & 308 & 209 & 84 \\
vvi-miR3623-5p & UCACAAGUUCAUCCAAGCACCA & 22 & 0 & 167 & 87 \\
vvi-miR3634-3p-1 & UUCCGACUCGCACUCAUGCCGU & 22 & 177 & 0 & 61 \\
vvi-miR396b & UUCCACAGCUUUCUUGAACU & 20 & 0 & 115 & 95 \\
vvi-miR396b-1 & UUCCACAGCUUUCUUGAACUU & 21 & 346 & 0 & 82 \\
vvi-miR396c & UUCCACAGCUUUCUUGAACUG & 21 & 441 & 1,751 & 101 \\
vvi-miR398b & UGUGUUCUCAGGUCGCCCCUG & 21 & 13 & 307 & 64 \\
vvi-miR403a & UUAGAUUCACGCACAAACUCG & 21 & 172 & 47 & 93 \\
vvi-miR482-5p & AAUUGGAGAGUAGGAAAGCUU & 21 & 0 & 529 & 109 \\
\hline
\end{tabular}

Target prediction identified 1,194 gene targets of miRNAs expressed in EC and 1,182 targets of the leaf miRNAs. From these, 104 enriched GO terms, which could be reduced to 52 clusters, were identified among the EC miRNA targets and 141 GO terms, summarised in 68 GO clusters for those of the leaf miRNAs. Network diagrams for the enriched GO clusters are shown in figure 1.
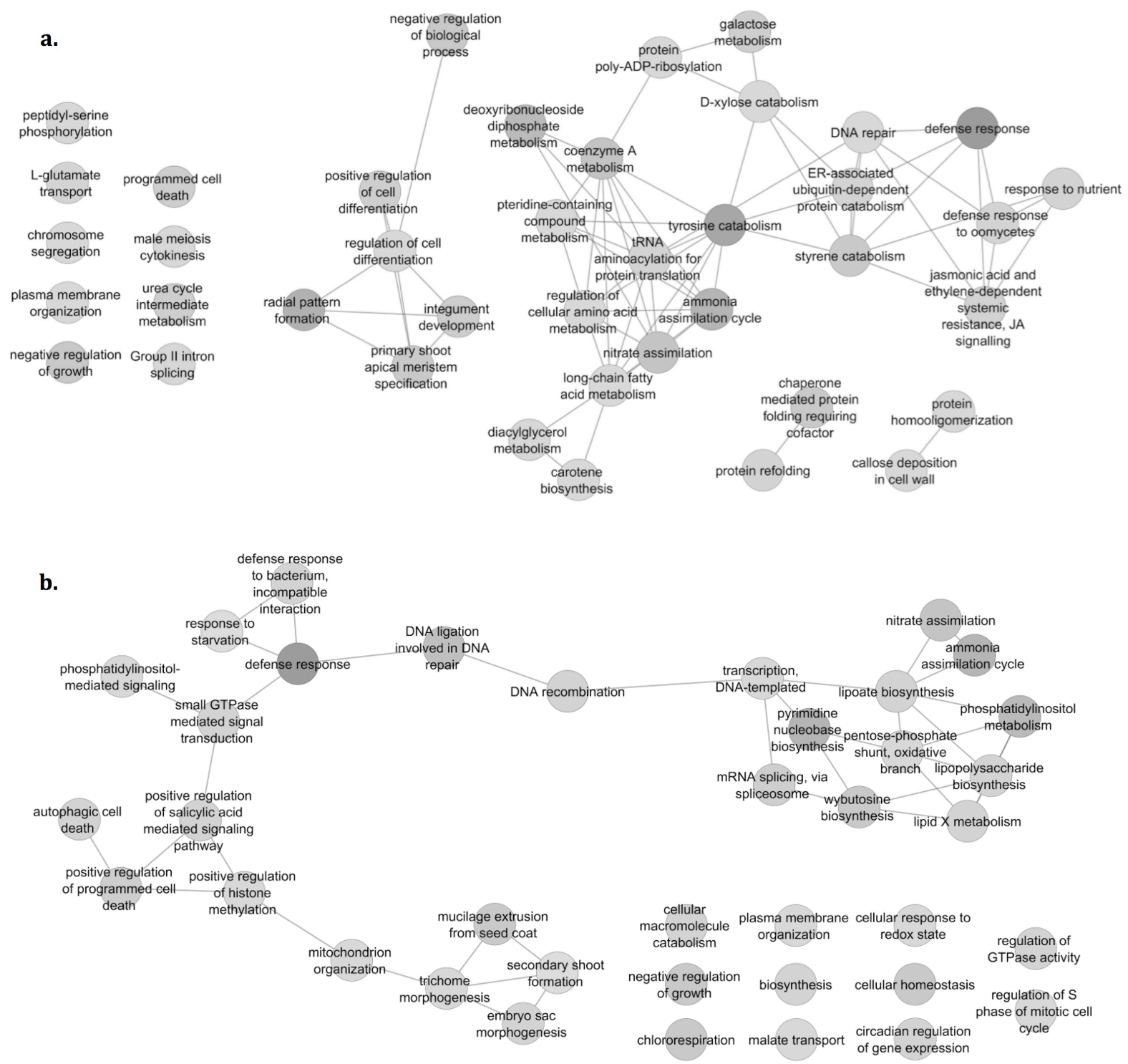
Figure 1. Enriched GO terms $(\mathrm{p}<0.05)$ for genes regulated by miRNAs expressed to over 100RPM in (a) young leaves and (b) embryogenic callus. Links indicate closely related GO term clusters. Darker shaded nodes indicate clustered GO terms more significantly enriched (lower REVIGO p-value).

Common targets are evident among the two miRNA regulatory networks, with defence response genes most highly enriched for both libraries. However, this is associated with response to oomycetes and jasmonic acid signalling in the case of leaf tissue and with response to bacterium, starvation and salicylic acid signalling in EC. Both target sets also include a group of biosynthesis and metabolism genes containing overlapping GO clusters. Of note among the EC miRNA targets are genes associated with positive regulation of histone modification, suggesting differential epigenetic regulation in this tissue. As expected, genes associated with vegetative tissue and apical meristem differentiation were identified among the targets of miRNAs from the leaf tissue and those associated with germination among targets of the EC miRNAs.

\section{Trans-acting siRNAs}

Following the removal of miRNA sequences from each read set, the remaining small RNA reads were grouped by size (Fig. 2). A higher proportion of EC siRNA fragments were 24 nt in length (55.5\%, compared with $26.4 \%$ in leaf), characteristic of heterochromatic siRNAs. In contrast, the leaf tissue showed a relatively higher proportion of $21 \mathrm{nt}$ siRNAs, which are associated with PTGS (in the case of tasiRNA) and RdDM of Pol II transcribed TEs.

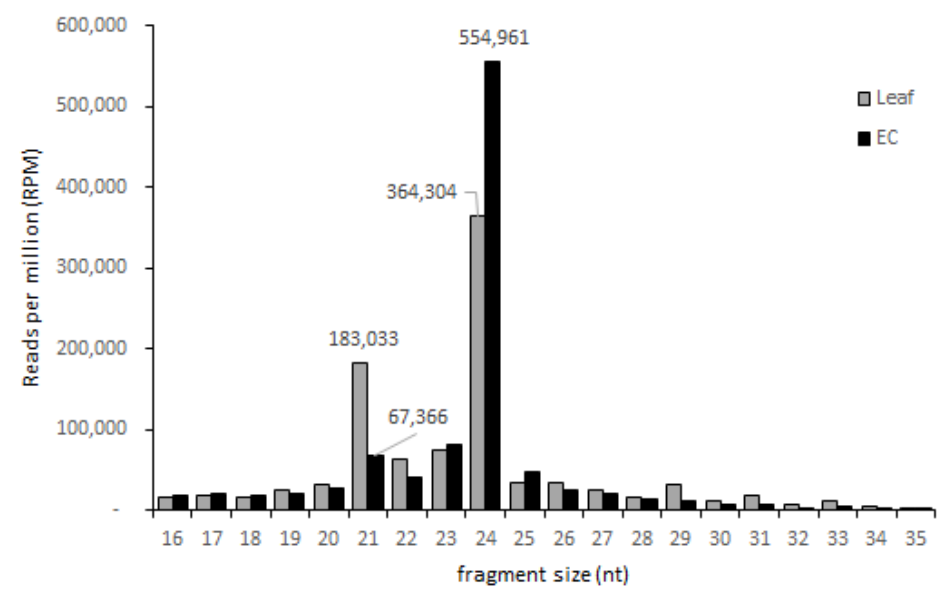

Figure 2. Small interfering RNA read abundance in grapevine young leaf and EC tissue by fragment size.

Trans-acting siRNA prediction revealed phased $21 \mathrm{nt}$ degradation products from 99 loci in the leaf sample, and from 82 loci in the EC sample. Due to highly uneven proportion of miRNA reads from each library (mir166 represents $0.33 \%$ of the leaf small RNA reads after filtering), tasiRNA reads were normalised to the total number of siRNA reads in millions.

Only two tasiRNA sequences were found at over 100RPM in the EC library. One sequence (TATGAGCTTCCATTTGACTGC) was 4.97-fold more abundant in leaf tissue, and the other (CTTCTTTCATTAGGGTTGCTT) was represented at similar levels (0.80-fold) in leaves. In contrast, of the 18 tasiRNA reads found at over 100RPM in leaf tissue, 14 were absent from the EC library and the remaining 4 were present at 4 -fold lower abundance or less. Therefore, tasiRNAs were only found that were elevated in the leaf tissue.

The previously identified TAS locus vvi-TAS4a / TAS7 on chromosome 14 (Rock, 2013; Zhang et al., 2012) was found to produce the highest number of phased tasiRNAs (6,603.9 RPM total) in the young leaf library. In the EC library, TAS4a did not pass the significance threshold for phased siRNA production. Previously characterised associations of tasiRNAs from the TAS4 locus in Arabidopsis with MYB 
transcription factors have led to the proposal that the TAS4 loci in grapevine may be involved in the regulation of bioflavonoid production (Rock, 2013).

Surprisingly, despite the high number of phased tasiRNAs from this loci, only 2 copies (0.6 RPM) of miR828a, the previously reported phase initiator for the TAS4 locus, were found among the leaf small RNA reads. No other 21-22 nt small RNA reads in the library predicted to initiate in-phase degradation of TAS4a transcripts within $200 \mathrm{bp}$ of the TAS4a phased siRNAs were found.

\section{CONCLUSIONS}

The small RNA profile of grapevine tissue maintained as embryogenic callus cultures is very different to that of young leaf vegetative tissue.

While microRNAs comprised as much as one third of the small RNA component of young leaf tissue, this is dominated by the presence of miR166, a conserved microRNA family that regulates the development of leaf structure and shoot apical meristem. In contrast, miRNAs comprised less that half of one per cent of EC small RNAs, with the most abundant sequences represented by miRNAs that regulate embryogenesis and the male germ line cells.

Heterochromatic siRNAs were approximately twice as abundant in EC tissue as leaf tissue, but tasiRNAs were not found to be enriched in this tissue. In contrast, 18 tasiRNAs were above threshold levels in leaf tissue, the most abundant of which were from the conserved TAS4a locus.

The dissimilarity between the small RNA profiles of EC and leaf tissue suggest that in addition to differences in gene regulation, it is likely that these two tissue types differ significantly with respect to epigenetic modifications of the DNA and possibly even the activity of endogenous TEs. It also appears that these two tissue types are primed for appreciably different responses to an environmental stress event or the presence of foreign DNA.

\section{ACKNOWLEDGEMENTS}

This research was funded by a joint initiative of New Zealand Wine, Lincoln University and Plant and Food Research New Zealand.

\section{Literature Cited}

Al-Shahrour, F., Díaz-Uriarte, R., and Dopazo, J. (2004). FatiGO: A web tool for finding significant associations of Gene Ontology terms with groups of genes. Bioinformatics 20, 578-580.

Chen, H.-M., Li, Y.-H., and Wu, S.-H. (2007). Bioinformatic prediction and experimental validation of a microRNA-directed tandem trans-acting siRNA cascade in Arabidopsis. Proc. Natl. Acad. Sci. U. S. A. 104, 3318-3323.

Dai, X., and Zhao, P.X. (2011). psRNATarget: A plant small RNA target analysis server. Nucleic Acids Res. 39, $155-159$.

Dhekney, S.A., Li, Z.T., Road, B., Compton, M.E., and Gray, D.J. (2009). Optimizing Initiation and Maintenance of Vitis Embryogenic Cultures. HortScience 44, 1400-1406.

Kidner, C. a, and Martienssen, R. a (2004). Spatially restricted microRNA directs leaf polarity through ARGONAUTE1. Nature $428,81-84$.

Kozomara, A., and Griffiths-Jones, S. (2014). MiRBase: Annotating high confidence microRNAs using deep sequencing data. Nucleic Acids Res. 42, 68-73.

Kuksova, V., Piven, N., and Gleba, Y. (1997). Somaclonal variation and in vitro induced mutagenesis in grapevine. Plant Cell. Tissue Organ Cult. 49, 17-27.

Lisch, D., and Bennetzen, J.J.L. (2011). Transposable element origins of epigenetic gene regulation. Curr. Opin. Plant Biol. $14,156-161$.

McCue, A.D., Nuthikattu, S., and Slotkin, R.K. (2013). Genome-wide identification of genes regulated in trans by transposable element small interfering RNAs. RNA Biol. 10, 1-17.

Meyers, B.C., Axtell, M.J., Bartel, B., Bartel, D.P., Bowman, J.L., Cao, X., Carrington, J.C., Chen, X., Pamela, J., Griffiths-jones, S., et al. (2016). Criteria for Annotation of Plant MicroRNAs.

Nawrocki, E.P., Burge, S.W., Bateman, A., Daub, J., Eberhardt, R.Y., Eddy, S.R., Floden, E.W., Gardner, P.P., Jones, T.A., Tate, J., et al. (2015). Rfam 12.0: Updates to the RNA families database. Nucleic Acids Res. 43, D130-D137.

Nodine, M.D., and Bartel, D.P. (2010). MicroRNAs prevent precocious gene expression and enable pattern formation during plant embryogenesis. Genes Dev. 24, 2678-2692.

Prüfer, K., Stenzel, U., Dannemann, M., Green, R.E., Lachmann, M., and Kelso, J. (2008). PatMaN: Rapid alignment of short 
sequences to large databases. Bioinformatics 24, 1530-1531.

Rock, C.D. (2013). Trans-acting small interfering RNA4: Key to nutraceutical synthesis in grape development? Trends Plant Sci. 18, 601-610.

Schellenbaum, P., Mohler, V., Wenzel, G., and Walter, B. (2008). Variation in DNA methylation patterns of grapevine somaclones (Vitis vinifera L.). BMC Plant Biol. 8, 78.

Shivaprasad, P. V, Chen, H., Patel, K., Bond, D.M., Santos, B.A.C.M., and Baulcombe, D.C. (2012). A MicroRNA Superfamily Regulates Nucleotide Binding Site - Leucine-Rich Repeats and Other mRNAs. Plant Cell 24, 859-874.

Slotkin, R.K., and Martienssen, R. (2007). Transposable elements and the epigenetic regulation of the genome. Nat. Rev. Genet. 8, 272-285.

Stocks, M.B., Moxon, S., Mapleson, D., Woolfenden, H.C., Mohorianu, I., Folkes, L., Schwach, F., Dalmay, T., and Moulton, V. (2012). The UEA sRNA workbench: A suite of tools for analysing and visualizing next generation sequencing microRNA and small RNA datasets. Bioinformatics 28, 2059-2061.

Supek, F., Bo??njak, M., ??kunca, N., and ??muc, T. (2011). REVIGO summarizes and visualizes long lists of gene ontology terms. PLoS One 6.

Torregrosa, L. (1998). A simple and efficient method to obtain stable embryogenic cultures from anthers of Vitis vinifera L. Vitis 37, 91-92.

Vitulo, N., Forcato, C., Carpinelli, E.C., Telatin, A., Campagna, D., D’Angelo, M., Zimbello, R., Corso, M., Vannozzi, A., Bonghi, C., et al. (2014). A deep survey of alternative splicing in grape reveals changes in the splicing machinery related to tissue, stress condition and genotype. BMC Plant Biol. 14, 99.

Voinnet, O. (2009). Origin, Biogenesis, and Activity of Plant MicroRNAs. Cell 136, 669-687.

Wu, X.-M., Liu, M.-Y., Ge, X.-X., Xu, Q., and Guo, W.-W. (2011). Stage and tissue-specific modulation of ten conserved miRNAs and their targets during somatic embryogenesis of Valencia sweet orange. Planta 233, 495-505.

Zhang, C., Li, G., Wang, J., and Fang, J. (2012). Identification of trans-acting siRNAs and their regulatory cascades in grapevine. Bioinformatics 28, 2561-2568. 